\title{
THz Spectroscopy of Extremely Shallow Acceptors States in Ge/GeSi Multiple-Quantum-Well Heterostructures
}

\author{
V.Ya. Aleshikin ${ }^{a}$, I.V. Erofeeva ${ }^{a}$, V.I. Gavrilenko ${ }^{a}$, \\ A.V. IkOnnikov ${ }^{a}$, D.V. KozLOV ${ }^{a}$, O.A. Kuznetsov ${ }^{a}$ \\ AND D.B. VEKSLER ${ }^{b}$ \\ ${ }^{a}$ Institute for Physics of Microstructures, GSP-105 \\ Nizhny Novgorod, 603950, Russia \\ ${ }^{b}$ Rensselaer Polytechnic Institute, Troy, NY, USA
}

\begin{abstract}
New shallow acceptor magnetoabsorption lines in $\mathrm{THz}$ range have been discovered under bandgap photoexcitation in strained Ge/GeSi multiple-quantum-well heterostructures. It is shown, both theoretically and experimentally, that the resonant absorption results from the photoionization of $\mathrm{A}^{+}$-centers and from $1 s \rightarrow 2 p_{+}$-type transitions from the ground state of the barrier-situated $\mathrm{A}^{0}$-centers into excited states in the 1st and 2nd electronic subbands. The shallowest discovered ground acceptor states $\left(E_{\mathrm{B}} \leq 0.5 \mathrm{meV}\right)$ are attributed to the "barrier-spaced" acceptors (a hole bound with an acceptor ion in the neighboring Ge quantum well).
\end{abstract}

PACS numbers: 71.55.Cn, 76.90.+d, 78.67.De

\section{Introduction}

Recent interest in the $\mathrm{THz}$ range has stimulated investigations of shallow impurities in strained $\mathrm{Ge} / \mathrm{Ge}_{1-x} \mathrm{Si}_{x}$ quantum well (QW) heterostructures [1-3]. The strain-induced valence band splitting in $\mathrm{Ge} / \mathrm{GeSi}$ structures decreases the effective masses of two-dimensional holes and the binding energies of confined acceptors compared with those in bulk Ge, in contrast to the donors whose binding energy is known to increase due to additional confinement of the wave function by the QW potential. In this paper, we employ the newly developed technique [3] to distinguish between different type shallow acceptor centers contributing to the magnetoabsorption in the $\mathrm{THz}$ range in $\mathrm{Ge} / \mathrm{GeSi}$ heterostructures. The method is based on measuring the differential impurity magnetoabsorption in $\mathrm{THz}$ range at the modulated bandgap optical excitation. The absorption signal is due to free 
carriers capture by ionized impurities that are always present in a sample because of a partial impurity compensation (cf. [1]). This method allows us to reveal complicated features of confined acceptor spectra not detected by conventional techniques.

\section{Experimental}

The structures under study were grown on $\mathrm{Ge}(111)$ substrate by vapor-phase epitaxy. Sample parameters are given in the captions to Figs. 1 and 2. The total thickness of the structures exceeds the critical one, thus leading to the stress relaxation in the interface structure-substrate. As a result Ge layers are biaxially compressed while GeSi layers are biaxially stretched. The residual shallow acceptor concentration was estimated to be $\sim 10^{14} \mathrm{~cm}^{-3}$ [1]. Magnetoabsorption of THz radiation ( 0.3 to $1.25 \mathrm{THz})$ generated by backward wave tubes (BWT) has been measured in a swept magnetic field (normal to the plane of the sample) at $T=$ $4.2 \mathrm{~K}$. Free carriers were excited by a modulated (usually $1 \mathrm{kHz}$ meander) bandgap light $(\lambda \sim 0.9 \mu \mathrm{m})$. The transmitted through the Ge/GeSi sample radiation has been detected by $n$-InSb crystal. To avoid the interference effects in the sample, the substrate has been polished to the wedge of $2^{\circ}$. A nearly circular polarization of the $\mathrm{THz}$ radiation has been employed to distinguish between the acceptor and donor absorption.

\section{Results and discussions}

Figure 1 shows the magnetoabsorption resonant line positions in sample \#306 with narrow Ge QWs. Two spectral lines $C H_{1}$ and $C h_{1}$ are known to result from cyclotron resonance $(\mathrm{CR})$ transitions between the lowest hole Landau levels in Ge QWs, $0 s_{1} \rightarrow 1 s_{1}$ and $3 a_{1} \rightarrow 4 a_{1}$ correspondingly [4]. In contrast to the CR lines, the positions of two other lines, $C I_{1}$ and $C I_{2}$, do not extrapolate to the origin of coordinates. Earlier we have observed the $\mathrm{CI}_{2}$ line in the impurity photoconductivity spectra [1,5] (see open symbols in Fig. 1) and attributed this line either to the photoionization of the $\mathrm{A}^{+}$-center (the $\mathrm{A}^{+}$-center is the $\mathrm{QW}$ acceptor ion binding two holes) or to the $1 s \rightarrow 2 p_{+}$transitions of the barrier situated neutral acceptors $\left(\mathrm{A}^{0}\right.$ - centers). The acceptor (rather than the donor) nature of the lines $C I_{1}$ and $C I_{2}$ is confirmed by the results of the absorption measurements with nearly circular polarized radiation. For uniformly distributed residual acceptors, only QW-centered neutral impurities (with the maximal binding energy about $7 \mathrm{meV}$ ) and the barrier-centered ones (with the minimal binding energy about $2 \mathrm{meV}$ ) give rise to the resonant absorption (cf. [6]). The transitions for QW situated acceptors are out of the spectral range accessible to BWT (they have been observed earlier in the photoconductivity measurements with Fourier transform spectrometer [1]).

To distinguish between $\mathrm{A}^{+}$-center and barrier situated $\mathrm{A}^{0}$-center absorption in the "low-frequency" spectral range, we calculated the energies of $1 s \rightarrow 2 p_{+}$ 


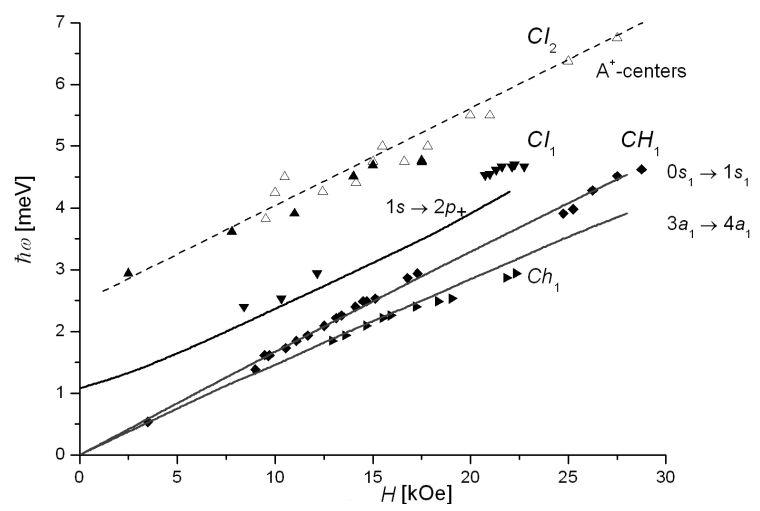

Fig. 1. Spectral positions of absorption (closed symbols) and photoconductivity (open symbols) $[1,5]$ resonances in the $\mathrm{Ge} / \mathrm{Ge}_{1-x} \mathrm{Si}_{x}$ sample \#306 $\left(x=0.12, d_{\mathrm{Ge}}=20 \mathrm{~nm}\right.$, $d_{\mathrm{GeSi}}=26 \mathrm{~nm}$, elastic deformation of Ge layers $\left.\varepsilon_{x x}=2.2 \times 10^{-3}, N_{\text {period }}=162\right)$. Solid lines - calculated $\omega(H)$ dependences for hole CR lines $C H_{1}$ and $C h_{1}$ and $1 s \rightarrow 2 p_{+}$ transitions for barrier-centered $\mathrm{A}^{0}$ acceptors $\left(C I_{1}\right)$. Dashed line - resonances attributed to photoionization of $\mathrm{A}^{+}$-centers $\left(C I_{2}\right)$.

transitions for the barrier situated $\mathrm{A}^{0}$-centers in the magnetic fields using the expansion of the acceptor wave function in terms of the hole wave functions in the Ge QW at $H=0$ (see Ref. [7] for details). Comparing the calculation results with the experimental data (see Fig. 1), we attributed the $C I_{1}$ line to the $1 s \rightarrow 2 p_{+}$transitions. The minor discrepancy (the experimental data for $C I_{1}$ line are slightly higher than the calculated magnetic field dependence for $1 s \rightarrow 2 p_{+}$ transitions) seems to be caused by dispersion of the transition energy for the uniformly distributed residual impurities. The $\mathrm{CI}_{2}$ line seems to result from the photoionization of the $\mathrm{A}^{+}$-centers. We estimated that the corresponding binding energy is $E_{+} \approx 2 \mathrm{meV}$ at $H=0$ [8] that is a little bit smaller than the value that $C I_{2}$ line cuts on the ordinate axis in Fig. 1. It is reasonable to assume that $\mathrm{A}^{+}$-center, just as a $\mathrm{D}^{-}$-center, has no excited states [9], so the observed optical transitions should take place at $\hbar \omega>E_{+}$.

Figure 2 presents a typical magnetoabsorption spectrum in the sample \#308 with broader Ge QW where three acceptor related lines $C I_{1}-C I_{3}$ are observed $[2,3]\left(C E_{1 \mathrm{~L}}\right.$ is $\mathrm{CR}$ line of $1 \mathrm{~L}$-electrons in GeSi layers [10]). The overall data on the spectral line positions are given in Fig. 3. Time-resolved measurements with pulsed bandgap optical excitation show that typical relaxation times for all impurity lines are as high as $10^{-4} \mathrm{~s}$ (Fig. 2). Therefore, these lines result from the optical transitions from the ground state of shallow acceptors rather than from their excited states (cf. [11]). Just as for sample \#306, the highest frequency line $\left(\mathrm{CI}_{3}\right)$ can be attributed to the photoionization of the $\mathrm{A}^{+}$-centers. The calculation results for the sample \#308 with broad QWs predict two optical transitions of comparable intensity from the ground $1 s$ state of barrier-situated $\mathrm{A}^{0}$-center into 


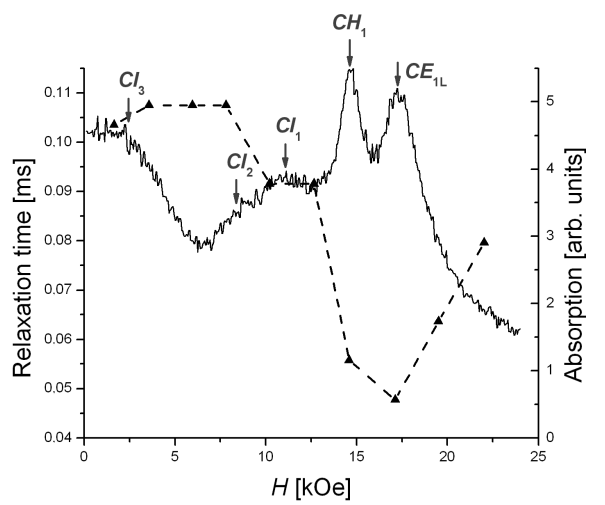

Fig. 2. Absorption (solid line) and signal decay time (symbols connected by a broken line) versus the magnetic field in the $\mathrm{Ge} / \mathrm{Ge}_{1-x} \mathrm{Si}_{x}$ sample \#308 $\left(x=0.09, d_{\mathrm{Ge}}=35 \mathrm{~nm}\right.$, $\left.d_{\mathrm{GeSi}}=16 \mathrm{~nm}, \varepsilon_{x x}=4.4 \times 10^{-4}, N_{\text {period }}=162\right)$ at the pulsed optical excitation $\left(\tau_{\text {pulse }}=330 \mu \mathrm{s}\right.$, repetition rate $\left.f=100 \mathrm{~Hz}\right) . \hbar \omega=2.43 \mathrm{meV}$.

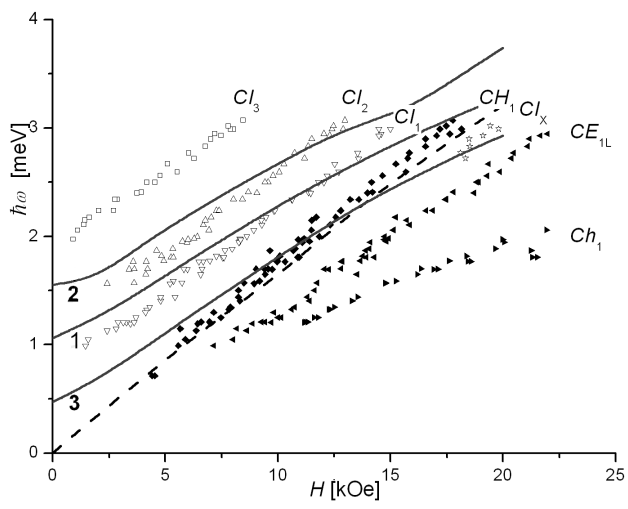

Fig. 3. Spectral positions of the observed absorption resonances in the sample \#308 (symbols). Dashed line - calculated $\omega(H)$ dependence for the hole $\mathrm{CR}$ line $\mathrm{CH}_{1}$. Solid lines 1 and 2 - calculated $\omega(H)$ dependences for transitions from $1 s$ state to $2 p_{+}$ states pertained to the 1 st and the 2 nd electric hole subband in the $\mathrm{QW}\left(C I_{1} \& C I_{2}\right)$ correspondingly. Black solid line 3 - calculated dependence for $1 s \rightarrow 2 p_{+}$transition for "barrier spaced" $\mathrm{A}^{0}$-center $\left(C I_{x}\right)$.

$2 p_{+}$states related to the 1 st and the 2 nd hole electronic subbands (Fig. 3 ). This results from a lower (compared to sample \#306) energy of the size quantization and from the state mixing in the 1 st and the 2 nd subbands.

In conclusion, we discovered a new acceptor related to magnetoabsorption line $C I_{x}$ that becomes discernible on a higher $H$ side of the main hole CR line $\mathrm{CH}_{1}$ when the frequency increased (see Fig. 3). We speculate that this line results from $1 s \rightarrow 2 p_{+}$transitions for very shallow "barrier-spaced" $\mathrm{A}^{0}$-center consisting of the hole bound with an acceptor ion in the neighboring Ge QW. According to 
our calculations (see Fig. 3), the energy of such transition only slightly exceeds that of the main hole CR transition in the magnetic fields up to $1 \mathrm{~T}$, and this line is typically masked by the powerful absorption line $C H_{1}$. However, at $H>17 \mathrm{kOe}$, this transition becomes distinguishable.

\section{Acknowledgment}

This work was financially supported by RFBR (Grants \#03-02-16808, \#04-02-17178), Russian Academy of Sciences and by Russian Ministry for Education and Science. The authors are thankful to M.D. Moldavskaya for collaboration, A.N. Panin and E.A. Uskova for technical assistance.

\section{References}

[1] V.I. Gavrilenko, I.V. Erofeeva, A.L. Korotkov, Z.F. Krasil'nik, O.A. Kuznetsov, M.D. Moldavskaya, V.V. Nikonorov, L.V. Paramonov, Pis'ma Zh. Eksp. Teor. Fiz. 65, 194 (1997) (JETP Lett. 65, 209 (1997)).

[2] V.Ya. Aleshkin, I.V. Erofeeva, V.I. Gavrilenko, A.V. Ikonnikov, D.V. Kozlov, O.A. Kuznetsov, D.B. Veksler, Physica B 340-342, 840 (2003).

[3] V.Ya. Aleshkin, A.V. Antonov, D.B. Veksler, V.I. Gavrilenko, I.V. Erofeeva, A.V. Ikonnikov, D.V. Kozlov, O.A. Kuznetsov, Fiz. Tverd. Tela 46, 126 (2004) (Sov. Phys. Solid State 46, 125 (2004)).

[4] V.Ya. Aleshkin, V.I. Gavrilenko, I.V. Erofeeva, O.A. Kuznetsov, M.D. Moldavskaya, V.L. Vaks, D.B. Veksler, in: Proc. 6th Int. Symp. Nanostructures: Physics and Technology, Ioffe Institute, St.Petersburg (Russia) 1999, p. 356.

[5] V.Ya. Aleshkin, B.A. Andreev, V.I. Gavrilenko, I.V. Erofeeva, D.V. Kozlov, O.A. Kuznetsov, M.D. Moldavskaya, A.V. Novikov, Physica E 7, 608 (2000).

[6] S. Huant, W. Knap, R. Stepniewski, G. Martinez, V. Thierry-Mied, B. Etienne, in: High Magnetic Fields in Semiconductor Physics II, Ed. G. Landwehr, in Springer Series in Solid-State Sciences, Vol. 87, Springer Verlag, Berlin 1989, p. 293.

[7] V.Ya. Aleshkin, A.V. Antonov, D.B. Veksler, V.I. Gavrilenko, I.V. Erofeeva, A.V. Ikonnikov, D.V. Kozlov, O.A. Kuznetsov, K.E. Spirin, in: Proc. Workshop on Nanophotonics, Institute for Physics of Microstructures, N. Novgorod (Russia) 2004, p. 129 (in Russian); Fiz. Tverd. Tela, accepted for publication.

[8] V.Ya. Aleshkin, V.I. Gavrilenko, D.V. Kozlov, in: Proc. Workshop on Nanophotonics, Institute for Physics of Microstructures, N. Novgorod (Russia) 2003, p. 318 (in Russian).

[9] A.B. Dzyubenko, Phys. Lett. A 165, 357 (1992).

[10] V.Ya. Aleshkin, D.B. Veksler, V.I. Gavrilenko, I.V. Erofeeva, A.V. Ikonnikov, D.V. Kozlov, O.A. Kuznetsov, Fiz. Tverd. Tela 46, 131 (2004) (Sov. Phys.-Solid State 46, 130 (2004)).

[11] S.V. Meshkov, E.I. Rashba, Zh. Eksp. Teor. Fiz. 76, 2206 (1979) (Sov. Phys.JETP 49, 1115 (1979)). 